\title{
PATIENT PERCEPTIONS REGARDING OUTPATIENT HIP AND KNEE ARTHROPLASTY
}

\author{
R. Michael Meneghini $\mathrm{MD}^{1,2}$ \\ Mary Ziemba-Davis BA ${ }^{2}$
}
${ }^{1}$ Indiana University School of Medicine, Department of Orthopaedic Surgery, $1120 \mathrm{~W}$.
Michigan Street, Room 600, Indianapolis, IN 46202
${ }^{2}$ Indiana University Health Physicians Orthopedics and Sports Medicine, IU Health Saxony Hospital, 13100 East $136^{\text {th }}$ Street, Suite 2000, Fishers, IN 46037

\section{Corresponding Author:}

R. Michael Meneghini, MD

Indiana University Health Physicians Orthopedics and Sports Medicine

Indiana University School of Medicine, Department of Orthopaedic Surgery

$13100136^{\text {th }}$ Street

Suite 2000

Fishers, IN 46037

Phone: 317-688-5980

Email: rmeneghi@iuhealth.org 


\section{PATIENT PERCEPTIONS REGARDING OUTPATIENT HIP AND KNEE ARTHROPLASTY}

\section{Abstract}

Introduction: There has been increasing interest in outpatient total joint arthroplasty (TJA) in the orthopedic community, but how patients feel about outpatient TJA is unknown. The purpose of this study was to understand patient perspectives on hip and knee replacements performed in an outpatient setting rather than an inpatient hospital.

Methods: We surveyed 110 consecutive patients scheduled for primary TJA in an academic suburban arthroplasty practice regarding their existing knowledge and perceptions of outpatient TJA. Questionnaires were administered during preoperative clinic visits prior to any discussion of surgery location or length of stay and prior to the preoperative joint replacement education class. No mention of outpatient surgery or same day discharge occurred prior to completion of the questionnaire.

Results: Fifty-seven percent of respondents were female, and $42.7 \%$ were age 65 or older.

Ninety-one percent of respondents had not previously had hip or knee replacement surgery.

Very few patients expected same-day discharge $(n=3)$ or even a one night stay in the hospital $(n$ $=17$ ). $54 \%$ expected to stay in the hospital two or more nights. Only $54.5 \%$ of patients were aware that outpatient TJA is an option, with $55.3 \%$ of men and $31.7 \%$ of women reporting they were comfortable or very comfortable with outpatient TJA under circumstances in which someone was available to assist them at home $(p=0.030)$. Conversely, $61 \%$ and $72.8 \%$, respectively, believed that faster recovery and decreased likelihood of hospital acquired infection are likely to be advantages of outpatient TJA. Interestingly, $51.9 \%$ of respondents felt ambulatory surgery centers are as safe as hospitals and $62.6 \%$ believed that home is the best place to recovery from TJA. 
26 Conclusion: These observations suggest there is both opportunity and need for patient

27 education regarding outpatient TJA. As outpatient hip and knee replacement becomes more

28 common, it is essential that patients understand the ambulatory surgery process, the benefits and

29 risks of same day discharge, and their role in a successful outpatient experience.

30 Keywords: outpatient knee arthroplasty, outpatient knee replacement, outpatient hip

31 arthroplasty, outpatient hip replacement, patient perspectives

32

33 


\section{Introduction}

There has been increasing interest in outpatient primary total hip (THA) and knee (TKA) arthroplasty.[1-10] The interest in outpatient arthroplasty has been fueled by financial considerations including the ability to reduce costs within the episode of care, surgeon ownership in ambulatory surgery centers (ASC), and the ability to more easily control the surgical care environment in an ASC.[1, 5, 8, 11]

Rapid recovery protocols have created a natural evolution from the inpatient to the outpatient setting.[2, 3, 6, 10, 12-14] Outpatient total joint arthroplasty (TJA) has been successfully performed during the past decade $[4,13,15-20]$, albeit for the most part by highly experienced surgeons with carefully selected patients. Success has been attributed to multidisciplinary care coordination, standardized perioperative protocols, discharge planning, and careful patient selection.[2-4, 7, 13, 15, 16] Although the feasibility and safety of outpatient TJA has been studied from the vantage point of the surgeon, to the best of our knowledge, no studies have investigated patient perspectives on outpatient TJA. The purpose of the study was to understand patient thoughts and perspectives on hip and knee replacements performed in an outpatient setting.

\section{Methods}

With institutional review board approval, all patients of the primary author scheduled for primary THA or TKA in an academic suburban arthroplasty practice that performs outpatient TJA were offered a 16-question survey regarding their existing knowledge and perceptions of outpatient TJA (Appendix A). Questionnaires were administered during the first preoperative clinic visits occurring between 11/01/2015 and 04/31/2016 prior to any discussion of surgery location or length of stay expectations and prior to the preoperative joint replacement education 
class. No mention of outpatient surgery or same day discharge occurred prior to completion of the questionnaire and the medical office did not contain any promotional material or information regarding outpatient, same-day, or rapid discharge total joint replacement. Patients were instructed not to put their names on the questionnaire to reduce potential bias associated with surgeon knowledge of their responses.

Statistical Analysis

Minitab 17 (State College, PA) was used for data analysis. Pearson's Chi-Square $\left(X^{2}\right)$ test was used to test independence among categorical variables with Fisher's $p$ reported for $2 \times 2$ tables. Content analysis was used to categorize open-ended, qualitative responses to survey questions 8 and 9.

\section{Results}

One hundred twelve patients were available for the study. Two declined the survey-one due to visual impairment and inability to read the questionnaire and one due to inability to read English. The final sample of 110 consecutive patient questionnaires represented a $98 \%$ response rate. Fifty-seven percent of respondents were female, and $11.8 \%$ were age 50 or younger $(\mathrm{n}=$ 13), $45.5 \%$ were age 51 to $64(n=50)$, and $42.7 \%$ were age 65 or older $(n=47)$. Respondents were scheduled for primary THA $(32.7 \%)$, TKA $(60.9 \%)$ or both $(6.4 \%)$. Ninety-one percent of respondents (98/108) had not previously had hip or knee replacement surgery. Patients who had previously undergone THA or TKA had done so in an inpatient setting. Ten of 44 respondents (22.7\%) had taken care of someone after hip or knee replacement surgery or assisted a patient who had outpatient TJA.

As shown in Table 1, only three respondents reported that they would expect to be discharged on the same day as TJA surgery. The majority (64.6\%) expected to be discharged in 
80

81

82

83

84

85

86

87

88

89

90

91

92

93

94

95

96

97

98

99

100

101

1 to 2 days, but one in five $(20.2 \%)$ thought they would be in the hospital three or more days

following TJA. Expectations for discharge between less than 24 hours and 1 day did not differ based on patient sex $\left(X^{2}=0.539, p=0.696\right)$ or type of scheduled joint replacement $\left(X^{2}=1.441\right.$. $p=0.249$ ). (Age group could not be tested due to small cell counts.)

When asked "Have you ever heard of outpatient joint replacement where patients are discharged home the day of surgery or within 23 hours of surgery?" $54.5 \%(n=60)$ responded yes. Awareness of outpatient joint replacement did not vary by sex $\left(X^{2}=1.479, p=0.248\right)$, age group $\left(X^{2}=0.297, p=0.862\right)$, or type of scheduled surgery $\left(X^{2}=0.077, p=0.962\right)$. Of the 60 patients who had heard about outpatient TJA, 53.3\% $(\mathrm{n}=32)$ had heard about it from family and friends, $18.3 \%(n=11)$ from a primary care physician, $16.7 \%(n=10)$ from an orthopedic surgeon, $3.3 \%(\mathrm{n}=2)$ from another patient, $1.7 \%(\mathrm{n}=1)$ from the internet, and $1.7 \%(\mathrm{n}=1)$

from television or radio. Five percent of respondents $(n=3)$ had heard about outpatient TJA from another source such as at work.

3 Survey respondents were asked "Assuming you have someone to assist you, how comfortable would you be being discharged the same day or within 23 hours of joint replacement surgery?" Overall, $13.0 \%$ (14/108) reported that they would be very comfortable, $21.3 \%$

$(23 / 108)$ reported that they would be comfortable, $33.3 \%(36 / 108)$ said they were unsure, $12.0 \%$ $(13 / 108)$ said they would be uncomfortable, and $20.4 \%(22 / 108)$ replied that they would be very uncomfortable with outpatient discharge following TJA. As shown in Figure 1, men were more comfortable with outpatient TJA surgery $\left(X^{2}=10.685, p=0.030\right)$ than women. Comfort level with outpatient surgery did not vary by age group $\left(X^{2}=6.538, p=0.587\right)$ or scheduled surgery type $\left(X^{2}=5.934, p=0.204\right)$ 

limitations of outpatient TJA - including limited implant types, use of the direct anterior

104

105

106

107

108

109

110

111

112

113

114

115

116

117

118 approach to THA, increased complications, difficult pain control, challenges regaining mobility, and challenging recovering from anesthesia - were unlikely. Sixty-one percent and $72.8 \%$, respectively, believed that faster recovery than expected and decreased likelihood of hospital acquired infection were likely to be advantages of outpatient TJA (Table 2). Perceptions of faster recovery did not vary by patient sex $\left(X^{2}=1.316, p=0.303\right)$ or scheduled surgery type $\left(X^{2}\right.$ $=2.868, p=0.238)$, but significantly more patients age 50 or younger $(83.3 \%)$ vs. $50 \%$ of those age 51 to 64 and $68.3 \%$ of those age 65 or older thought faster recovery was a likely advantage of outpatient TJA $\left(X^{2}=5.889, p=0.053\right)$. Significantly more women $(80.7 \%)$ than men $(62.2 \%)$ believed that outpatient surgery would reduce the likelihood of hospital acquired infection $\left(X^{2}=\right.$ 4.312, $p=0.046)$. Perspectives on hospital acquired infection in general did not vary based on age group $\left(X^{2}=0.106, p=0.949\right)$ or type of scheduled surgery $\left(X^{2}=1.393, p=0.498\right)$.

Overall, $36.8 \%(39 / 106)$ of respondents felt it was safer to have TJA surgery in a hospital, $11.3 \%(12 / 106)$ felt that ASCs are safer, and 51.9\% (55/106) felt that hospitals and ASCs are equally safe (Figure 2$)$. This observation did not vary by patient $\operatorname{sex}\left(X^{2}=0.926, p=\right.$ $0.629)$ or scheduled surgery type $\left(X^{2}=0.719, p=0.698\right)$. Reflecting a statistical trend, $20 \%$ of respondents between the ages of 51 and 64 compared to none of those age 50 or younger and $4.7 \%$ of those age 65 or older reported that ASCs are safer than hospitals $\left(X^{2}=8.132, p=0.087\right)$. Most respondents $(62.6 \%, 67 / 107)$ believed it would be better to recover from joint replacement surgery at home. Twenty-three percent (25/107) believed recovering in the hospital would be better, and 14\% (15/107) did not think that recovery would be better in one place or the other (Figure 3). Perceptions of recovery at home or in the hospital did not vary based on patient 
125

sex $\left(X^{2}=1.279, p=0.528\right)$, age group $\left(X^{2}=5.488, p=0.241\right)$, or scheduled surgery type $\left(X^{2}=\right.$ 0.032, $p=0.984)$.

Patients were asked about optimal conditions for outpatient TJA, including whether it is appropriate for partial or total joint replacement and what makes someone a good or a poor candidate for outpatient TJA. Thirty-nine percent of respondents (40/102) reported that outpatient surgery is most appropriate for partial joint replacement, 5\% (5/102) felt it was most appropriate for total joint replacement, and 55.9\% (57/102) felt it was appropriate for both partial and total joint replacement. Table 3 presents responses to open-ended questions asking what factors or characteristics would make someone a good or a poor candidate for outpatient joint replacement surgery. Respondents uniformly identified the presence or absence of overall good health, care support and/or a safe home environment, positive attitude/outlook/motivation, pain management, obesity, and age as important contributing factors.

\section{Discussion}

Outpatient TJA is on the rise in the United States due to increasing emphasis on reducing the cost of healthcare and the ability for surgeons to more readily control surgical care in ASCs. The increase in outpatient TJA has been possible due to significant advances in the perioperative management of joint replacement patients including multidisciplinary care coordination, standardized perioperative protocols, discharge planning, and careful patient selection.[2-4, 7, 13, 15, 16] To date, however, patient knowledge of and opinions on outpatient hip and knee replacement are unknown. This descriptive study was conducted to enhance understanding of patient views on TJA performed in an outpatient setting rather than in an inpatient hospital. In our convenience sample of consecutive patients scheduled for hip and/or knee replacement, 54\% expected to stay in the hospital two or more days following surgery, only three 
respondents reported they would not expect to stay in the hospital overnight, and only 17 reported that they expected to be discharged after one night in the hospital, suggesting that same day and early discharge were far from common patient expectations. Slightly more than half of respondents had heard of outpatient joint replacement defined as being discharged the day of surgery or within 23 hours of surgery. Awareness of outpatient joint replacement did not vary based on patient sex, age group (50 or younger, 51 to 64 , and 65 or older), or scheduled surgery type (THA, TKA, or both). Most respondents had heard about outpatient TJA from family or friends $(53.3 \%)$. Thirty-five percent had heard about it from a primary care physician or orthopedic surgeon.

Only $11.7 \%$ of women compared to $31.9 \%$ of men reported that they were very comfortable being discharged the same day or within 23 hours of joint replacement surgery, a statistically different observation $(p=0.030)$. Comfort level with outpatient TJA did not vary by age group or type of scheduled TJA. The majority of patients reported that potential perceived limitations of outpatient joint replacement - including limited implant types, use of the direct anterior approach to THA, increased complications, difficult pain control, challenges regaining mobility, and challenging recovering from anesthesia - were unlikely. Conversely, a majority believed that faster recovery than expected - especially those age 50 or younger $(p=0.053)$-and decreased likelihood of hospital acquired infection - especially women $(p=0.046)$-- were likely to be advantages of outpatient TJA. Slightly more than half of all respondents felt that total joint replacement was equally safe in hospitals and ASCs. Independent of sex, age group, and type of scheduled surgery, two out of three respondents believed it would be better to recover from joint replacement at home rather than in the hospital. Respondents identified the presence or absence of overall good health, care support and/or a safe home environment, 
171

positive attitude/outlook/motivation, pain management, obesity, and age as factors which would determine whether someone is a good or a poor candidate for outpatient TJA.

Our study is limited by the use of a convenience sample of patients scheduled for total hip and/or knee replacement with a single surgeon in a single arthroplasty practice.

Nevertheless, the study was conducted to acquire the first, initial insight into patient perspectives on outpatient TJA. It is hoped that it will be instigate similar inquiries in diverse TJA settings, especially those offering outpatient hip and knee replacement surgery. As outpatient hip and knee replacement becomes more common, it will be important to ensure that patients understand the ambulatory surgery process, the benefits and risks of same day discharge, and their role in a successful outpatient experience. Fulfillment of these objectives should be predicated on solid patient understanding of outpatient TJA. Hunt et al.[21] observed that patients may not overtly state concerns about early discharge following THA due, in part, to their role as patients dependent upon the expertise of healthcare providers. In addition, patient expectations regarding length of hospital stay may reflect unstated needs and motivations such as concerns about burdening family members with their care. These represent important additional areas for research-based understanding and clinical communication.

Overall, our observations suggest that there is ample opportunity and need for patient education on the topic of outpatient joint replacement. Prior to preoperative education, very few patients scheduled for TJA expected zero nights, or even a one night stay, in the hospital. Two or more nights in the hospital was the predominant expectation. One-half of patients were not even aware that outpatient TJA is an option. Only 1 in two male patients and 3 in 10 female patients reported being comfortable or very comfortable with outpatient joint replacement under circumstances in which someone was available to assist them at home, despite the fact that most 
194 patients felt ASCs were as safe as hospitals and that home is the best place to recovery from 195 TJA. 
196

197

198

199

200

201

202

203

204

205

206

207

208

209

210

211

212

213

214

215

216

217

218

\section{References}

1. Aynardi M, Post Z, Ong A, Orozco F, Sukin DC. Outpatient surgery as a means of cost reduction in total hip arthroplasty: a case-control study. HSS journal : the musculoskeletal journal of Hospital for Special Surgery 10(3): 252, 2014

2. Berger RA. A comprehensive approach to outpatient total hip arthroplasty. American journal of orthopedics 36(9 Suppl): 4, 2007

3. Berger RA, Jacobs JJ, Meneghini RM, Della Valle C, Paprosky W, Rosenberg AG. Rapid rehabilitation and recovery with minimally invasive total hip arthroplasty. Clinical orthopaedics and related research (429): 239, 2004

4. Berger RA, Sanders S, Gerlinger T, Della Valle C, Jacobs JJ, Rosenberg AG. Outpatient total knee arthroplasty with a minimally invasive technique. The Journal of arthroplasty 20(7 Suppl 3): 33,2005

5. Bertin KC. Minimally invasive outpatient total hip arthroplasty: a financial analysis. Clinical orthopaedics and related research (435): 154, 2005

6. Dorr LD, Thomas DJ, Zhu J, Dastane M, Chao L, Long WT. Outpatient total hip arthroplasty. The Journal of arthroplasty 25(4): 501, 2010

7. Kolisek FR, McGrath MS, Jessup NM, Monesmith EA, Mont MA. Comparison of outpatient versus inpatient total knee arthroplasty. Clinical orthopaedics and related research 467(6): 1438, 2009

8. Lovald S, Ong K, Lau E, Joshi G, Kurtz S, Malkani A. Patient selection in outpatient and short-stay total knee arthroplasty. Journal of surgical orthopaedic advances 23(1): 2, 2014 9. Sculco PK, Pagnano MW. Perioperative solutions for rapid recovery joint arthroplasty: get ahead and stay ahead. The Journal of arthroplasty 30(4): 518, 2015 
10. Stambough JB, Nunley RM, Curry MC, Steger-May K, Clohisy JC. Rapid recovery protocols for primary total hip arthroplasty can safely reduce length of stay without increasing readmissions. The Journal of arthroplasty 30(4): 521, 2015

11. Lavernia CJ, Villa JM. Rapid recovery programs in arthroplasty: the money side. The Journal of arthroplasty 30(4): 533, 2015

12. Hozack WJ, Matsen-Ko L. Rapid recovery after hip and knee arthroplasty: a process and a destination. The Journal of arthroplasty 30(4): 517, 2015

13. Berger RA, Kusuma SK, Sanders SA, Thill ES, Sporer SM. The feasibility and perioperative complications of outpatient knee arthroplasty. Clinical orthopaedics and related research 467(6): 1443,2009

14. Berend KR, Lombardi AV, Jr., Mallory TH. Rapid recovery protocol for peri-operative care 230 of total hip and total knee arthroplasty patients. Surgical technology international 13: 239, 2004

231 15. Berger RA, Sanders S, D'Ambrogio E, Buchheit K, Deirmengian C, Paprosky W, Della Valle CJ, Rosenberg AG. Minimally invasive quadriceps-sparing TKA: results of a comprehensive pathway for outpatient TKA. The journal of knee surgery 19(2): 145, 2006 rehabilitation protocols enable outpatient hip replacement in selected patients. Clinical orthopaedics and related research 467(6): 1424, 2009 
242 19. Klein GR, Posner JM, Levine HB, Hartzband MA. Same Day Total Hip Arthroplasty

243 Performed at an Ambulatory Surgical Center: 90-Day Complication Rate on 549 Patients. The

244 Journal of arthroplasty, 2016

245 20. Springer BD, Odum SM, Vegari DN, Mokris JG, Beaver WB, Jr. Impact of Inpatient Versus

246 Outpatient Total Joint Arthroplasty on 30-Day Hospital Readmission Rates and Unplanned

247 Episodes of Care. The Orthopedic clinics of North America 48(1): 15, 2017

248 21. Hunt GR, Hall GM, Murthy BVS, O’Brien S, Beverland D, Lynch MC, Salmon P. Early

249 discharge following hip arthroplasty: patients' acceptance masks doubts and concerns. Health

250 Expectations 12(2): 130, 2009

251 
The authors thank Ethan Meneghini for conscientious data entry and project assistance. 
Table 1: Patient Expectations for Length of Hospital Stay After TJA

\begin{tabular}{|l|c|c|}
\hline & n & \% \\
\hline Less than 24 hours & 3 & 3.0 \\
\hline Less than 24 hours to 1 day & 4 & 4.0 \\
\hline 1 to 2 days & 64 & 64.6 \\
\hline 2 to 3 days & 8 & 8.1 \\
\hline$\geq 3$ days & 20 & 20.2 \\
\hline Total & 99 & 100.0 \\
\hline
\end{tabular}

Six respondents provided answers that could not be categorized (1 to 7 days, as few as possible, etc.). Four respondents did not answer the question. 


\begin{tabular}{|l|c|c|c|}
\hline Table 2: Patient Perspectives on Perceived Advantages and Disadvantages of Outpatient TJA \\
\hline & $\mathbf{N}$ & $\begin{array}{c}\text { \% } \\
\text { Responding } \\
\text { Likely }\end{array}$ & $\begin{array}{c}\text { \% } \\
\text { Responding } \\
\text { Unlikely }\end{array}$ \\
\hline Limit the type of implant I can receive & 104 & 29.8 & 70.2 \\
\hline Keep my doctor from using the direct anterior approach & 88 & 26.1 & 73.9 \\
\hline Result in a complication that I might not otherwise have had & 102 & 29.4 & 70.6 \\
\hline Help me recover faster than expected & 101 & 61.4 & 38.6 \\
\hline Make my pain harder to control & 103 & 32.0 & 68.0 \\
\hline Interfere with my ability to regain mobility & 103 & 21.4 & 78.6 \\
\hline Reduce the chance that I will get a hospital-acquired infection & 103 & 72.8 & 27.2 \\
\hline Make recovering from anesthesia more difficult & 101 & 17.8 & 82.2 \\
\hline
\end{tabular}




\begin{tabular}{|c|c|c|c|c|}
\hline \multirow[b]{2}{*}{ Characteristic } & \multicolumn{2}{|c|}{$\begin{array}{l}\text { Good Candidate for } \\
\text { Outpatient TJA }\end{array}$} & \multicolumn{2}{|c|}{$\begin{array}{l}\text { Poor Candidate for } \\
\text { Outpatient TJA }\end{array}$} \\
\hline & $\begin{array}{l}\text { No. Reporting } \\
\text { Characteristic }\end{array}$ & $\begin{array}{c}\text { \% Reporting } \\
\text { Characteristic }\end{array}$ & $\begin{array}{l}\text { No. Reporting } \\
\text { Characteristic }\end{array}$ & $\begin{array}{l}\text { \% Reporting } \\
\text { Characteristic }\end{array}$ \\
\hline Good/poor overall health & 47 & $43.9 \%$ & 48 & $46.2 \%$ \\
\hline $\begin{array}{l}\text { Presence/absence of care support and } \\
\text { safe home environment }\end{array}$ & 32 & $29.9 \%$ & 30 & $28.8 \%$ \\
\hline Managed/unmanaged pain & 21 & $19.6 \%$ & $\begin{array}{r}23 \\
\end{array}$ & $22.1 \%$ \\
\hline $\begin{array}{l}\text { Presence/absence of positive } \\
\text { attitude/outlook/motivation }\end{array}$ & 20 & $18.7 \%$ & 15 & $14.4 \%$ \\
\hline Younger/older age & 15 & $14.0 \%$ & 12 & $11.5 \%$ \\
\hline Not obese/obese & 10 & $9.3 \%$ & 11 & $10.6 \%$ \\
\hline
\end{tabular}




\section{Figure Legends}

Figure 1: Same day discharge comfort level by sex

Figure 2: Distribution of survey responses on safest location for surgery.

Figure 3: Distribution of survey responses on optimal location to recover from joint replacement surgery 


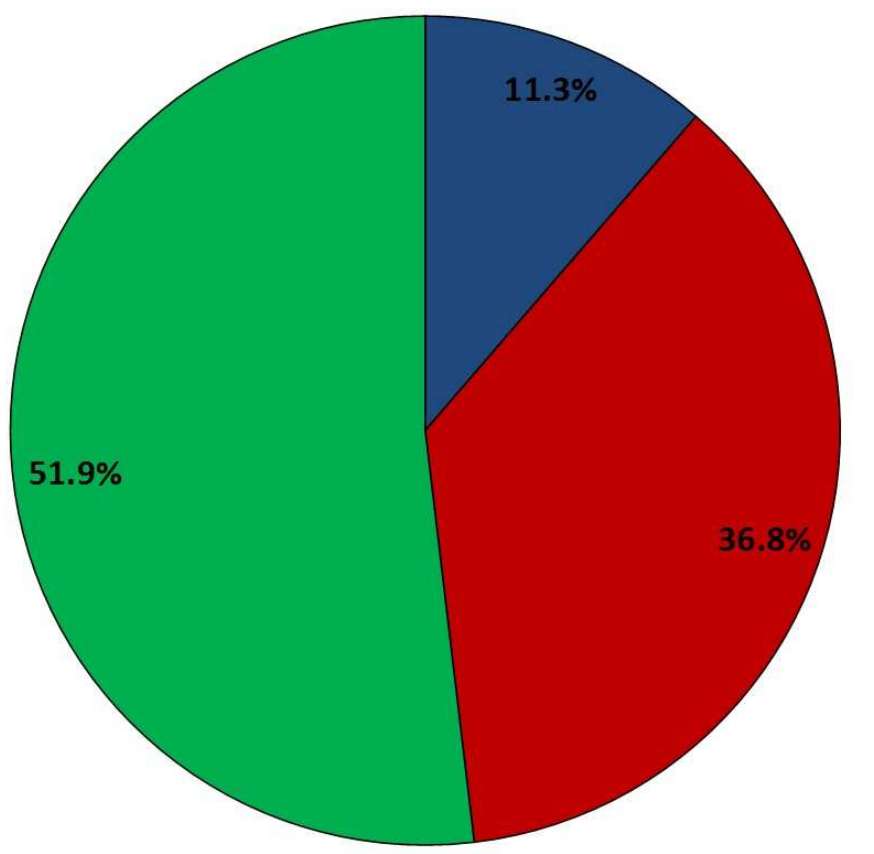

- Ambulatory Surgery Centers are Safer

- Hospitals are Safer

$\square$ ASCs and Hospitals are Equally Safe 


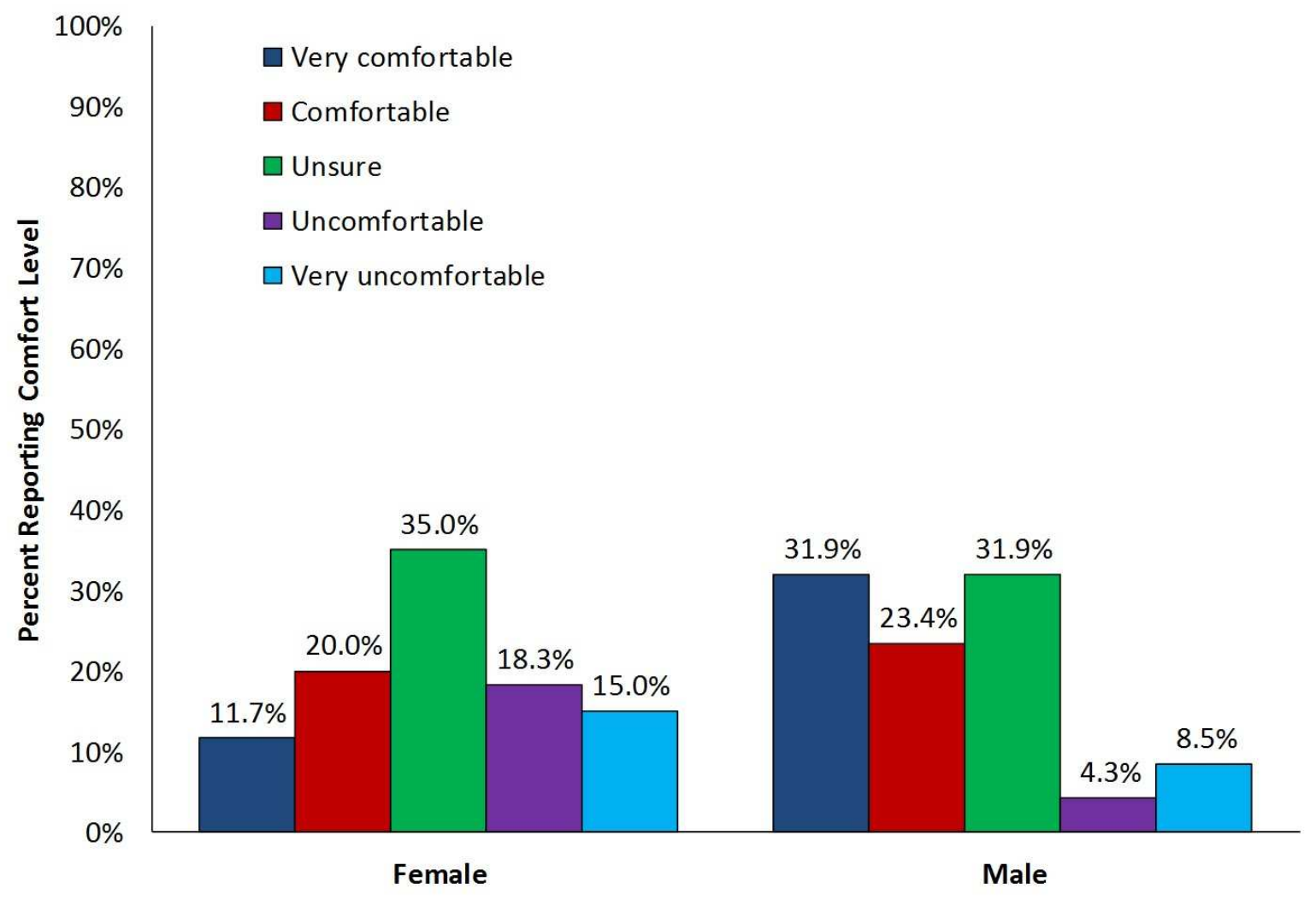




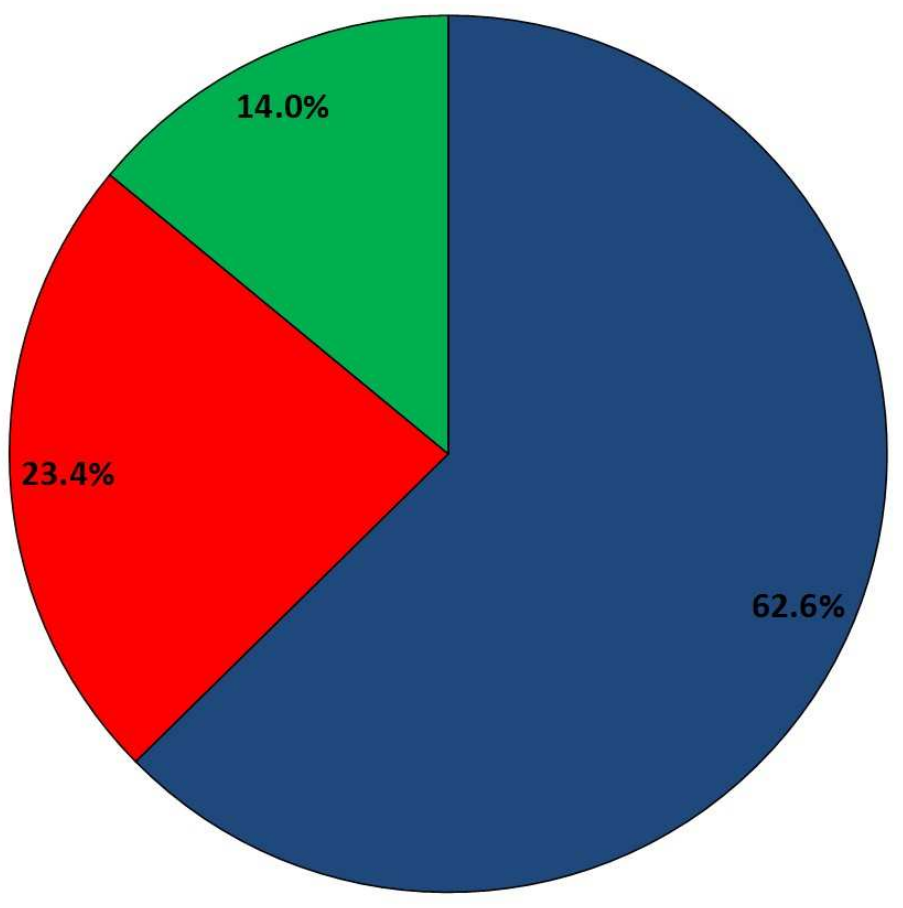

$\square$ At Home $\quad \square$ In the Hospital $\quad \square$ No Difference 
PATIENT VIEWS ON OUTPATIENT JOINT REPLACEMENT

1. Gender (circle one): Male

Female

2. Age (circle one): 50 or less

$51-64$

65 or greater

3. Are you here today for a hip or a knee problem?

Hip Knee Both

4. In general, how many nights would you expect to stay in the hospital after joint replacement surgery?

5. Have you ever heard of outpatient joint replacement where patients are discharged home the day of surgery or within 23 hours of surgery (circle one answer)?

Yes (please go to question 6) No (please go to question 7)

6. Where or from whom did you hear about outpatient joint replacement? (check all that apply):

Family or friends

Another patient

Your family or primary care doctor

TV or radio

Other (please specify):
Newspaper

Orthopaedic surgeon

Internet

7. Assuming you have someone to assist you, how comfortable would you be being discharged the same day or within 23 hours of joint replacement surgery (circle one answer)?

Very uncomfortable Uncomfortable Unsure Comfortable Very comfortable

8. In your opinion, what factors or characteristics would make someone a good candidate for outpatient joint replacement surgery?

9. What would make someone a poor candidate for outpatient joint replacement?

10. Please indicate whether you think the following statements are likely or unlikely (circle one answer for each statement).

\begin{tabular}{|l|r|r|}
\hline OUTPATIENT JOINT REPLACEMENT MIGHT.... & & \\
\hline ...Limit the type of implant I can receive & Likely & Unlikely \\
\hline $\begin{array}{l}\text {...Keep my doctor from being able to use the direct anterior } \\
\text { approach }\end{array}$ & Likely & Unlikely \\
\hline \begin{tabular}{l}
...Result in a complication that I might not otherwise have had \\
\hline
\end{tabular} & Likely & Unlikely \\
\hline
\end{tabular}




\begin{tabular}{|l|r|r|}
\hline ...Help me recover faster than expected & Likely & Unlikely \\
\hline ...Make my pain harder to control & Likely & Unlikely \\
\hline ...Interfere with my ability to regain mobility & Likely & Unlikely \\
\hline ...Reduce the chance that I will get a hospital-acquired infection & Likely & Unlikely \\
\hline ...Make recovering from anesthesia more difficult & Likely & Unlikely \\
\hline
\end{tabular}

11. Do you think that outpatient surgery would be appropriate for partial joint replacements, total joint replacements, or both (circle one answer)?

Partial joint replacements Total joint replacements Both

12. In your view, is it safer to have surgery in an ambulatory surgery center (ASC) or in a hospital?
ASC's are safer
Hospitals are safer
They are equally safe

13. In general, do you believe it would be better for you to recover from joint replacement at home or in the hospital?
At Home
In the Hospital
No difference

14. Have you had hip or knee replacement surgery (circle one answer)?

Yes - If yes, was it inpatient or outpatient surgery (circle answer)?

No

15. Have you taken care of someone after they had hip or knee replacement surgery (circle one answer)?

Yes - If yes, did they have outpatient surgery (circle answer)? $\quad$ Yes $\quad$ No No

16. Please feel free to add any additional comments you may have here:

You are finished with the survey. Thank you for your time. 